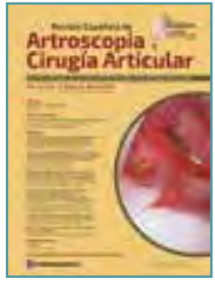

Vol. 25. Fasc. 1. Núm. 61. Marzo 2018

ISSN: 2386-3129 (impreso)

2443-9754 (online)

\title{
Originales
}

\section{Incidencia de calcificaciones heterotópicas tras artroscopia de cadera con técnica fuera-dentro}

\author{
X. Albillos, AD. Cuéllar, J. Martínez, R. Cuéllar
}

Servicio de Cirugía Ortopédica y Traumatología.

Hospital Universitario Donostia. San Sebastián

Correspondencia:

Dr. Xabier Albillos Sánchez

Correo electrónico: xabieralbillos@gmail.com
Recibido el 11 de septiembre de 2017

Aceptado el 3 de enero de 2018

Disponible en Internet: marzo de 2018

\section{RESUMEN}

Objetivo: conocer la incidencia de calcificaciones heterotópicas $(\mathrm{CH})$ tras artroscopia de cadera mediante técnica outside-inside. Comparación con incidencias publicadas en otros estudios sobre calcificaciones en la artroscopia de cadera.

Métodos: se realizó una revisión retrospectiva de las caderas intervenidas desde enero de 2012 hasta junio de 2016, mediante artroscopia de cadera por choque femoroacetabular (CFA) con técnica outside-inside. De los 119 casos totales, excluyendo caderas previamente intervenidas 0 aquellas intervenidas por otra causa que no fuese CFA, obtuvimos un total de 106 casos para el estudio. Se dispone de radiografías de la cadera intervenida de forma preoperatoria y postoperatoria con un tiempo mínimo de 2 meses desde la intervención. Se ha tomado como referencia la clasificación de Brooker.

Resultados: durante este periodo, 106 casos cumplen criterios de inclusión. La tasa de $\mathrm{CH}$ en nuestros pacientes ha sido globalmente de un $8 \%$. Si estratificamos los resultados según profilaxis o no con antiinflamatorios no esteroideos (AINE) de forma postoperatoria obtuvimos una tasa de calcificaciones del $6 \%$ en el grupo AINE frente a una tasa del 14\% en el grupo no AINE. En el análisis estadístico las diferencias observadas no han sido estadísticamente significativas $(p=0,123)$. Todas las calcificaciones fueron Brooker I o II.

\section{ABSTRACT \\ Incidence of heterotopic ossification after hip arthroscopy with outside-inside technique}

Objective: to know the incidence of heterotopic ossifications after hip arthroscopy using an outside-inside technique. Comparison of the incidence published in other studies on ossifications due to hip arthroscopy.

Methods: we performed a retrospective review of the hips operated by arthroscopy for FAl (femoroacetabular impingement) with outside-inside technique from January 2012 to June 2016. Excluding previously operated hips or those operated for other than FAl causes, we obtained a total of 106 cases. Radiographs were obtained preoperatively and postoperatively with a minimum time of 2 months from the intervention.

Results: during this period 119 patients were treated by hip arthroscopy, of whom 106 met inclusion criteria. The rate of heterotopic calcifications in our patients has been $8 \%$ globally. If we stratified the results according to prophylaxis or not with nonsteroidal anti-inflammatory drugs (NSAID), we obtained a rate of calcifications of $6 \%$ in the NSAID group and a rate of $14 \%$ in the non-NSAID group. All calcifications were Brooker I or II.

Conclusions: we have obtained results concordant to previous studies. The rate of heterotopic calcifications in 
Conclusiones: nuestros resultados son acordes con los de otros estudios sobre la tasa de $\mathrm{CH}$ en la artroscopia de cadera, siendo dicha tasa baja y menor que en la cirugía abierta. Por tanto, la cirugía artroscópica de cadera es un tratamiento seguro en este sentido. El tratamiento con AINE disminuye la tasa de calcificaciones, aunque esta diferencia no ha sido estadísticamente significativa en el presente artículo, si bien en la literatura ya se ha mostrado su efecto protector. En nuestra experiencia, la tasa de calcificaciones no aumenta de forma significativa por el empleo de la técnica fuera-dentro respecto a la vía clásica.

Nivel de evidencia: III.

Relevancia clínica: la artroscopia de cadera mediante técnica fuera-dentro para el tratamiento del CFA tiene una incidencia de CH similar a la de la técnica clásica.

Palabras clave: Calcificación heterotópica. Artroscopia. Cadera.

\section{Introducción}

La aparición de calcificaciones heterotópicas ( $\mathrm{CH}$ ) tras la cirugía artroscópica de cadera es una complicación que, aunque rara, puede comprometer los resultados de nuestra indicación quirúrgica. Las publicaciones relativas a la tasa de esta complicación tras cirugía abierta son numerosas. Dicha tasa es bien conocida, especialmente tras artroplastia total de cadera ${ }^{(1-4)}$, pero la incidencia de dicha complicación tras artroscopia de cadera aún no está tan caracterizada(5-8). En su mayoría son artículos que hacen referencia a casos de artroscopia de cadera realizados mediante abordaje clásico, siendo escasas las publicaciones que traten sobre la incidencia de $\mathrm{CH}$ tras cirugía artroscópica mediante técnica fuera-dentro(9).

La tasa reportada de $\mathrm{CH}$ en la cirugía abierta de cadera varía según los estudios, estando descrita una tasa de hasta el $90 \%^{(1)}$. De entre estos estudios, destaca un artículo de revisión de Neal, donde recogen los resultados de 218 estudios, con más de 51.000 cirugías de cadera, en el cual se publica que la incidencia de $\mathrm{CH}$ es de un $43 \%$ tras artroplastia de cadera(4). En cuanto a la cirugía artroscópica de cadera, se ha descrito una tasa del 0 al $12 \%$ de $\mathrm{CH}^{(5,6,6,7,9)}$. Según las publicaciones esta tasa puede alcanzar un $44 \%$ si no se prescriben antiinflamatorios no esteroideos (AINE) como arthroscopic hip surgery is lower than in open surgery, NSAID treatment decreases the rate of such calcifications and therefore arthroscopic hip surgery is a safe treatment in this sense. We did not find a statistically protective effect of NSAIDs, but there are several studies published that do so. In our experience, the ossification rate does not increase significantly by the use of the outside-inside technique in relation to the classical approach.

Level of evidence: III.

Clinical relevance: hip arthroscopy using an outside-inside technique for the treatment of FAl has an incidence of heterotopic calcifications similar to that of the classical approach.

Key words: Heterotopic ossification. Hip. Arthroscopy.

profilaxis postoperatoria(1,10,11).

La CH es la formación de hueso estructuralmente normal y maduro donde no debe aparecer, en los tejidos blandos ${ }^{(5)}$. La clasificación más extendida para las $\mathrm{CH}$ de cadera es la de Brooker, en la cual hay 4 grados (Tabla 1). El grado I sería un islote aislado en las partes blandas. El grado II sería aquella $\mathrm{CH}$ de origen acetabular o femoral con $>1 \mathrm{~cm}$ de separación entre superficies óseas. En el grado III habría $<1 \mathrm{~cm}$ de separación entre superficies. El grado IV ya sería un puente óseo (anquilosis).

Como hemos descrito previamente, dichas calcificaciones, en caso de ser Brooker I o II, son calcificaciones de pequeño tamaño que posiblemente no ocasionen repercusión clínica alguna al paciente. En cambio, si se trata de calcificaciones grandes (Brooker III O IV), estas pueden ser cau-

\section{Tabla 1. Clasificación de Brooker de calcificaciones heterotópicas}

\begin{tabular}{l|l}
\hline Grado I & Islote aislado en partes blandas \\
\hline Grado II & $\begin{array}{l}\text { Origen acetabular o femoral con }>1 \mathrm{~cm} \text { de } \\
\text { separación entre superficies óseas }\end{array}$ \\
\hline Grado III & $\begin{array}{l}\text { Origen acetabular o femoral con }<1 \mathrm{~cm} \mathrm{de} \\
\text { separación }\end{array}$ \\
\hline Grado IV & Anquilosis/Puente óseo \\
\hline
\end{tabular}


sa de pérdida del rango de movilidad y dolor. En caso de que sean sintomáticas se pueden plantear diferentes opciones de tratamiento, desde el conservador al quirúrgico, habiendo en dicho caso un alto riesgo de recidiva.

El objetivo de este artículo es describir la tasa de $\mathrm{CH}$ presente en nuestros pacientes tras la realización de artroscopia de cadera por técnica fuera-dentro para el tratamiento de un choque femoroacetabular (CFA), pudiéndolo así comparar con las incidencias publicadas en la literatura de casos intervenidos con la técnica de abordaje clásica. Asimismo, caracterizar cómo son dichas calcificaciones de acuerdo a la clasificación de Brooker y la relación con la toma o no de tratamiento profiláctico con AINE, ya que no está clara la necesidad de protocolizar dicho tratamiento en el postoperatorio. Para ello, hemos realizado una revisión retrospectiva de los casos intervenidos en estos últimos años.

\section{Métodos}

Realizamos una revisión retrospectiva de datos recogidos prospectivamente de los pacientes tratados quirúrgicamente de artroscopia de cadera por CFA desde enero de 2012 a junio de 2016, realizados por el mismo cirujano, mediante técnica fuera-dentro (CAM, PINCER o mixta), con un total de 106 casos (93 pacientes). En todos los casos se realizó una radiografía de pelvis anteroposterior preoperatoria y una radiografía postoperatoria tras al menos 2 meses desde la cirugía.

Como criterio de inclusión se ha tenido en cuenta toda artroscopia de cadera realizada en ese periodo de tiempo, que haya sido tratada por un CFA y que tuviese un control radiológico preoperatorio y postoperatorio con un mínimo de 2 meses tras la cirugía. Los pacientes han sido intervenidos mediante técnica fuera-dentro, con tracción de la extremidad para la fase acetabular. En su mayoría han sido pacientes tratados por un CFA de carácter mixto, con lesión labral. En caso de presencia de calcificación, tal y como se ha realizado en otros estudios, la hemos clasificado según la clasificación de Brooker para CH (Figura 1).

Los criterios de exclusión han sido, por un lado, pacientes previamente intervenidos de dicha cadera, bien haya sido por cirugía abierta o artroscópica. Por otro lado, pacientes en los que se haya rea-

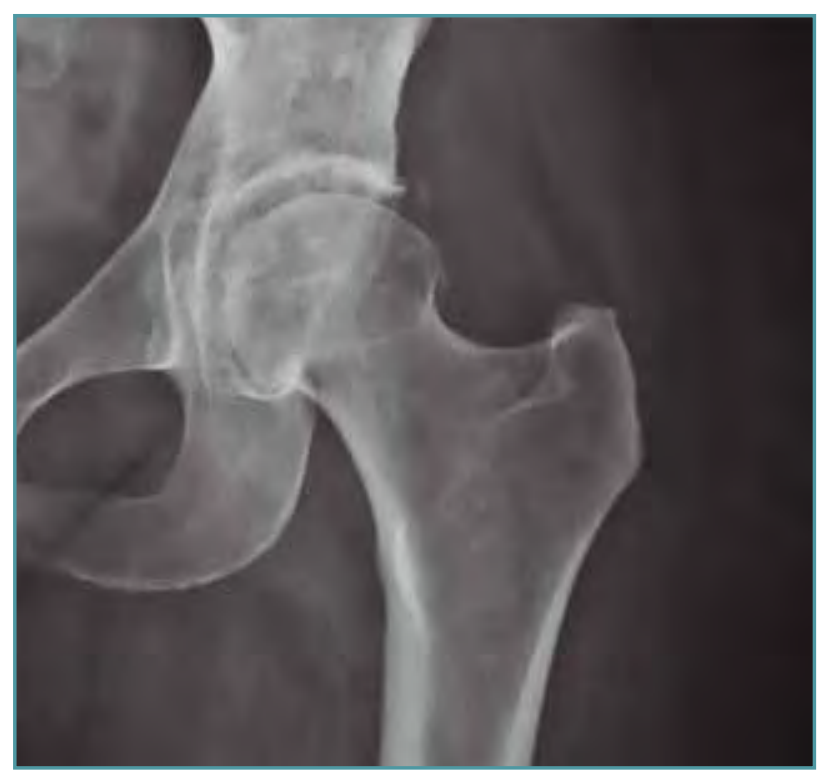

Figura 1. Radiografía anteroposterior de cadera. Control a los 3 meses de la cirugía con presencia de calcificación heterotópica (Brooker I).

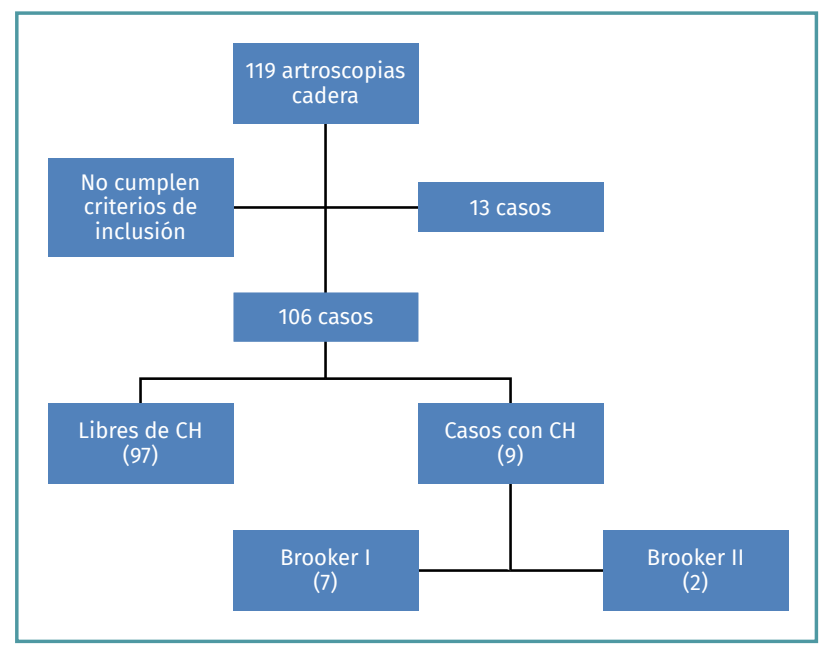

Figura 2. Selección de pacientes. CH: calcificaciones heterotópicas.

lizado artroscopia de cadera por otro motivo, como cadera en resorte, condromatosis sinovial, trocanteritis o tendinitis de psoas. También han sido descartados aquellos pacientes en los que no hay control radiológico más allá de los 2 meses de la cirugía. De los 119 casos intervenidos en este periodo de tiempo 13 han sido excluidos por ello, quedando por tanto 106 casos para el estudio (Figura 2).

De los pacientes incluidos en el estudio también hemos evaluado la presencia de tratamien- 


\section{Tabla 2. Pacientes con presencia de calcificaciones heterotópicas (CH). Clasificación de Brooker, edad del paciente y tratamiento analgésico/ antiinflamatorio postoperatorio recibido}

\begin{tabular}{c|c|c|c}
\hline Paciente & Brooker & Edad & Profilaxis \\
\hline 1 & II & 27 & No \\
\hline 2 & I & 49 & No \\
\hline 3 & II & 50 & No \\
\hline 4 & I & 39 & No \\
\hline 5 & I & 28 & Sí \\
\hline 6 & I & 36 & Sí \\
\hline 7 & I & 42 & Sí \\
\hline 8 & II & 37 & Sí \\
\hline 9 & I & 35 & Sí \\
\hline
\end{tabular}

to antiinflamatorio, ya que en los casos más antiguos no estaba protocolizado el empleo de AINE, con el fin de corroborar la bibliografía presente en torno al efecto protector de estos para las calcificaciones. Desde 2013 venimos empleando tratamiento antiinflamatorio. Desde finales de 2014 el tratamiento empleado en nuestros pacientes es el celecoxib a dosis de 200 mg por día durante 21 días, excepto en casos de alergia o intolerancia.

\section{Resultados}

Entre enero de 2012 y junio de 2016 se realizaron un total de 119 artroscopias de cadera, de las cuales cumplían los criterios de inclusión 106 (93 pacientes). Dichos pacientes han sido tratados por CFA mediante artroscopia de cadera con técnica fuera-dentro. Los pacientes tenían una edad media de 38 años en el momento de la intervención, con un rango que oscila de los 18 a los 59 años. De estos, el $71 \%$ de los pacientes eran hombres y el $29 \%$ eran mujeres.

La última radiografía postoperatoria se obtuvo en promedio 6 meses después de la operación, con un rango de entre 2 y 50 meses. La tasa global de $\mathrm{CH}$ en nuestro estudio fue del 8\% (9 casos en 106 caderas). De estos casos de $\mathrm{CH}, 7$ casos fue- ron clasificados como Brooker I y 2 casos como Brooker II (Tabla 2), lo cual es un dato más a favor de la literatura que presenta que las calcificaciones tras artroscopia de cadera son generalmente pequeñas, no siendo fuente de clínica ni de pérdida de rango de movilidad.

Como se ha mencionado anteriormente, durante 2012 y hasta marzo de 2013 no empleábamos AINE como protocolo de profilaxis, por lo que tenemos parte de nuestros pacientes que no recibieron tratamiento antiinflamatorio y sabemos que esto es importante para la incidencia de $\mathrm{CH}$. Es por ello que hemos decidido separar aquellos pacientes que han recibido tratamiento antiinflamatorio de aquellos que no y ver por tanto la tasa de calcificaciones estratificando por la toma de AINE.

Cuando se analizaron los datos según la profilaxis con AINE frente a analgésicos, se obtuvo una incidencia del $14 \%$ ( 4 casos en 25 pacientes) de $\mathrm{CH}$ para los pacientes que no tomaban AINE y una incidencia del $6 \%$ ( 5 casos en 81 pacientes) de $\mathrm{CH}$ para los que los toman (Figura 3). Hemos analizado dichos datos estadísticamente, mediante el test de chi cuadrado de Pearson $(p=0,123)$ y el test exacto de Fisher $(p=0,210)$, al tratarse de una comparación de proporciones, siendo las diferen-

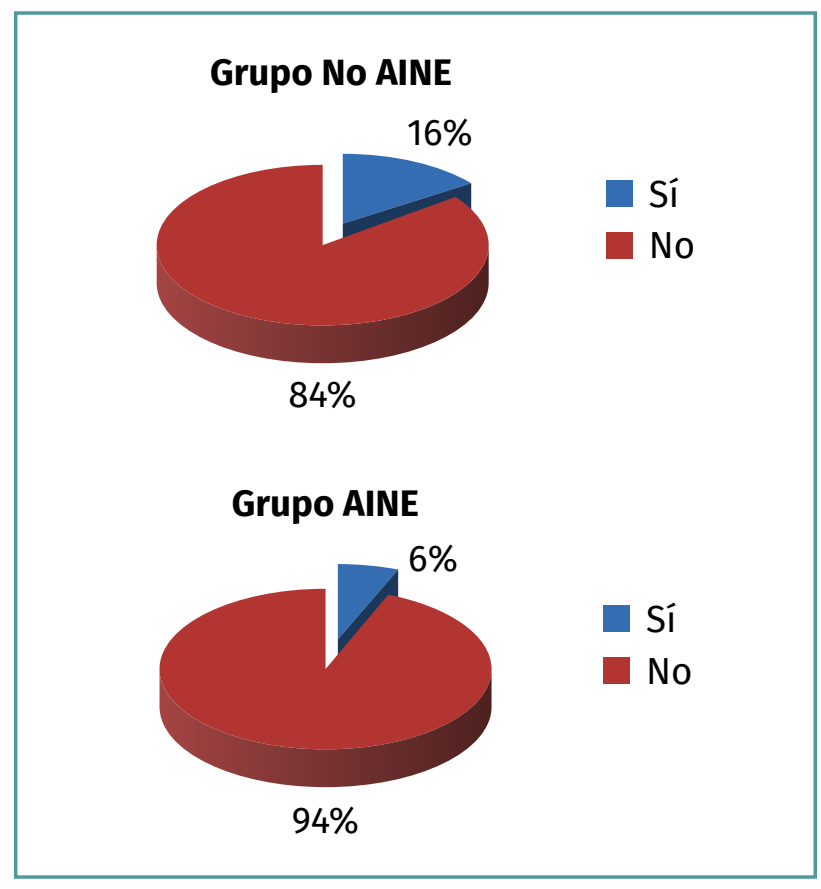

Figura 3. Incidencia de calcificaciones en función de la profilaxis con antiinflamatorios no esteroideos (AINE). 


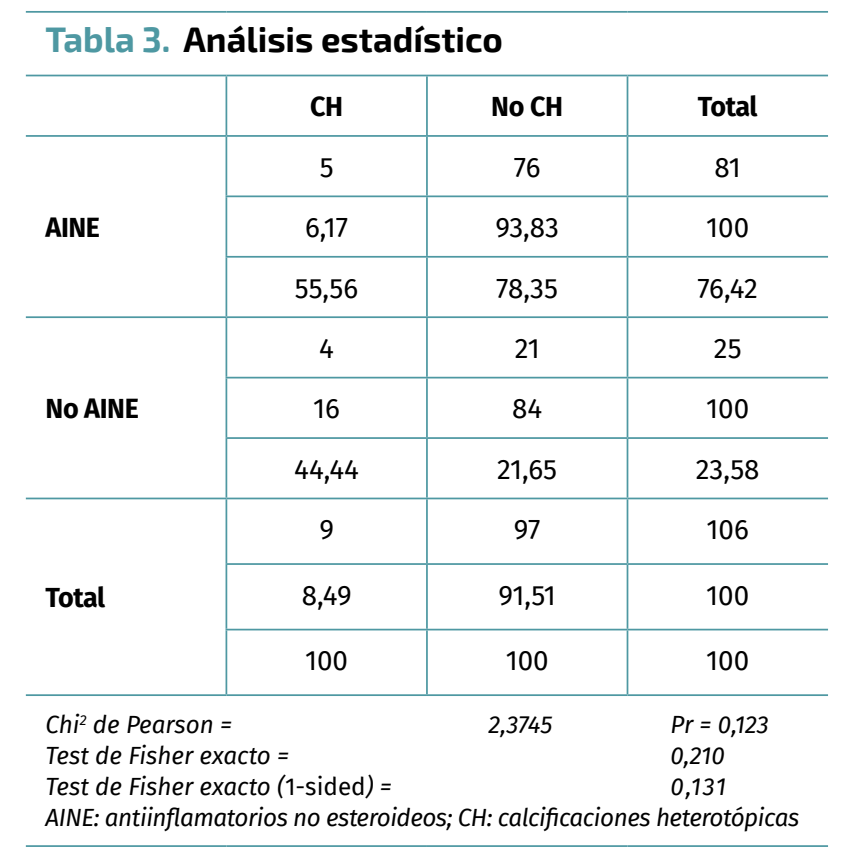

cias observadas no estadísticamente significativas (Tabla 3). Por lo que en nuestro estudio no podemos concluir que los AINE tengan un efecto protector para evitar la presencia de $\mathrm{CH}$. Es probable que habiendo tenido un tamaño muestral mayor el resultado hubiese sido diferente.

\section{Discusión y conclusiones}

Revisando la documentación publicada en PubMed en torno a la incidencia de $\mathrm{CH}$ tras artroscopia de cadera por CFA, hemos encontrado 10 estudios que la revisan. Además, hay escasa bibliografía en torno a la incidencia de estas tras abordaje fuera-dentro en la artroscopia de cadera, habiendo encontrado relativo a ello un artículo $^{(9)}$. En nuestro conocimiento, dicha diferencia en la incidencia, entre la técnica fuera-dentro y la técnica clásica, ha sido solo descrita por Sandoval, llegando en su estudio a la conclusión de que la tasa de calcificaciones es estadísticamente superior en la técnica fuera-dentro, siendo pequeña la magnitud de la diferencia.

En la bibliografía se ha descrito una tasa del 0 al $12 \%$ de $\mathrm{CH}$ tras la artroscopia de cadera ${ }^{(1,5,7,7)}$. Dicha tasa puede incluso alcanzar un $44 \%$ si no se prescriben AINE como profilaxis postoperatoria ${ }^{(1,10)}$.

La tasa descrita de $\mathrm{CH}$ en la literatura oscila del 0 al $12 \%$. Nuestra tasa de $\mathrm{CH}$, que es del $6 \%$ con profilaxis antiinflamatoria, está dentro de las tasas descritas en otros estudios, en su mayoría tratándose de cirugía artroscópica de cadera por abordaje clásico.

Las limitaciones de nuestro estudio son varias: por un lado, se trata de un estudio retrospectivo, con lo cual el análisis de datos sirve para extraer menor cantidad de conclusiones. Por otro lado, hemos tenido que subdividir los pacientes en torno a la toma o no de antiinflamatorios, ya que en los primeros casos no se aplicaba técnica profiláctica alguna, siendo la cantidad de pacientes que no han recibido tratamiento antiinflamatorio baja, lo cual puede tener relación con que las diferencias encontradas no hayan sido estadísticamente significativas. También conviene destacar que habría sido interesante que todos los pacientes que tomaban AINE hubiesen recibido el mismo fármaco en el postoperatorio de cara a evitar posibles sesgos. Por último, sabemos que las $\mathrm{CH}$ son multifactoriales, por lo que hay más factores que probablemente afectan los resultados.

También hay cierta controversia, ya reflejada en la literatura, relacionada con que la mayoría de las calcificaciones son pequeñas, Brooker de tipo I o II, las cuales, por tanto, es posible que clínicamente no produzcan ningún sintoma. Es por ello que hay autores que incluso se vuelven a cuestionar la necesidad de pautar antiinflamatorios como tratamiento profiláctico en el postoperatorio de la cirugía artroscópica de cadera ${ }^{(6,12)}$. Los posibles efectos secundarios del tratamiento con AINE deben tenerse en cuenta: intolerancia, alergia, ulcus gástrico, insuficiencia renal aguda (IRA), etc. En general, se respeta que dicha profilaxis es coste-efectiva en la artroscopia de cadera del adulto joven ${ }^{(12)}$.

Nuestros resultados son acordes con los de otros estudios sobre la tasa de $\mathrm{CH}$ en la artroscopia de cadera, siendo dicha tasa baja y menor que en la cirugía abierta. Por tanto, la cirugía artroscópica de cadera es un tratamiento seguro en este sentido.

El tratamiento con AINE disminuye la tasa de calcificaciones, aunque esta diferencia no ha sido estadisticamente significativa en el presente artículo, si bien en la literatura ya se ha mostrado su efecto protector.

En nuestra experiencia, la tasa de calcificaciones no aumenta de forma significativa por el empleo de la técnica fuera-dentro respecto a la vía clásica. 


\section{Responsabilidades éticas}

Protección de personas y animales. Los autores declaran que para esta investigación no se han realizado experimentos en seres humanos ni en animales.

Confidencialidad de los datos. LOS autores declaran que han seguido los protocolos de su centro de trabajo sobre la publicación de datos de pacientes.

Derecho a la privacidad y consentimiento informado. Los autores declaran que en este artículo no aparecen datos de pacientes.

Financiación. Los autores declaran no haber realizado gasto monetario alguno para la realización de este estudio.

Conflicto de interés. Los autores declaran no tener ningún conflicto de intereses.

\section{Bibliografía}

1. Kantor SR, Cummins J, Tanzer M. Complications after Total Hip Arthroplasty: Heterotopic Ossification. Semin Arthro. 2005;16:105-13.

2. Amar E, Sharfman ZT, Rath E. Heterotopic ossification after hip arthroscopy. J Hip Preserv Surg. 2015;2(4):355-63.

3. McCarthy EF, Sundaram M. Heterotopic ossification: a review. Skeletal Radiol. 2005;34:609-19.

4. Neal B, Gray H, MacMahon S, Dunn L. Incidence of heterotopic bone formation after major hip surgery. ANZ J Surg. 2002;72:808-21.
5. Rath E, Sherman H, Sampson TG, Ben Tov T, Maman E, Amar E. The Incidence of Heterotopic Ossification in Hip Arthroscopy. Arthroscopy. 2013 Mar;29(3):427-33.

6. Løken S. Hip Arthroscopy and Heterotopic Ossification: Is NSAID Prophylaxis Justified? Commentary on an article by James T. Beckmann, MD, MS, et al.: "Effect of Naproxen Prophylaxis on Heterotopic Ossification Following Hip Arthroscopy: A Double-Blind Randomized Placebo-Controlled Trial". J Bone Joint Surg. 2015;97(24):e80.

7. Larson CM, Giveans MR. Arthroscopic management of femoroacetabular impingement: early outcomes measures. Arthroscopy. 2008;24:540-6.

8. Bedi A, Zbeda RM, Bueno VF, Downie B, Dolan M, Kelly BT. The incidence of heterotopic ossification after hip arthroscopy. Am J Sports Med. 2012;40:854-63.

9. Sandoval E, Martín-Ríos MD, Cimas D, Masegosa A, Calvo E. Hip arthroscopy for the treatment of femoroacetabular impingement: a comparative study between the classic and the outside-in access. Hip Int. 2016 May 16;26(3):290-4.

10. Amar E, Sharfman ZT, Rath E. Heterotopic ossification after hip arthroscopy. J Hip Preserv Surg. 2015 Aug 28;2(4):355-63.

11. Randelli F, Pierannunzii L, Banci L, Ragone V, Aliprandi A, Buly R. Heterotopic ossifications after arthroscopic management of femoroacetabular impingement: the role of NSAID prophylaxis. J Orthop Traumatol. 2010 Dec;11(4):245-50.

12. Beckmann JT, Wylie JD, Potter MQ, Maak TG, Greene TH, Aoki SK. Effect of Naproxen Prophylaxis on Heterotopic Ossification Following Hip Arthroscopy: A Double-Blind Randomized Placebo-Controlled Trial. J Bone Joint Surg Am. 2015 Dec 16;97(24):2032-7. 